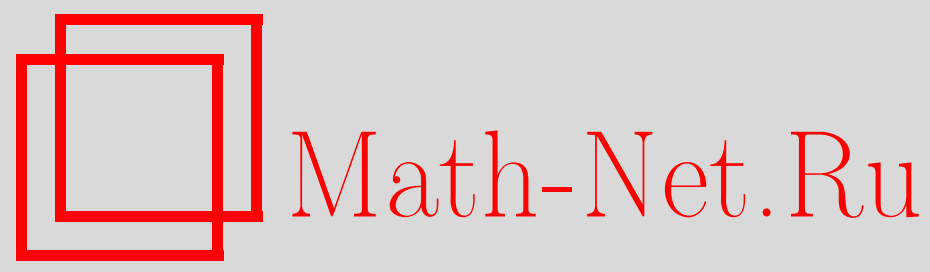

М. А. Гирнык, Приближение субгармонической в полуплоскости функции логарифмом модуля аналитической функции, Матем. заметки, 2005, том 78, выпуск 4, 483-492

DOI: https://doi.org/10.4213/mzm2610

Использование Общероссийского математического портала Math-Net.Ru подразумевает, что вы прочитали и согласны с пользовательским соглашением http://www.mathnet.ru/rus/agreement

Параметры загрузки:

IP: 34.229 .108 .108

26 апреля 2023 г., 17:00:04

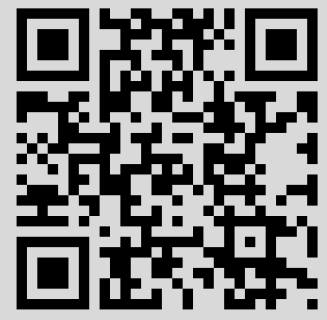




\section{ПРИБЛИЖЕНИЕ СУБГАРМОНИЧЕСКОЙ \\ В ПОЛУПЛОСКОСТИ ФУНКЦИИ ЛОГАРИФМОМ МОДУЛЯ АНАЛИТИЧЕСКОЙ ФУНКЦИИ}

\section{М. А. Гирнык}

Получено приближение в равномерной метрике вне исключительного множества функции, субгармонической в открытой полуплоскости, логарифмом модуля аналитической в полуплоскости функции для случаев конечного порядка и бесконечного нижнего порядка, а также оценка величины исключительного множества. Показано, что в случае конечного порядка найденная точность приближения существенно не улучшаема.

Библиограффия: 9 названий.

Результаты о приближении субгармонической функции логарифмом модуля аналитической имеют многочис ленные приложения в теории распределения значений, так как построить субгармоническую функцию с требуемьми асимптотическими свойствами зачастую значительно проще, чем аналитическую функцию. Главньм образом в связи с этим возникает задача приближения элементов более широкого множества субгармонических функций элементами из меньшего множества аналитических функций. Впервые в общем виде в классе субгармонических в плоскости функций конечного порядка эту проблему исследовал Азарин [1]. В 1985 году такоеприближение для произвольных субгармонических функций в произвольных областях рассмотрено в статье Юлмухаметова [2], где, в частности, найдена предельно точная аппроксимация для случая плоскости $\mathbb{C}$. Исследования Юлмухаметова продолжены и развиты в разных направлениях рядом авторов (см. обзор в [3], а также недавнюю работу [4]).

Здесь мы изучаем вьшеуказанное приближение в равномерной метрике для случая полуплоскости $\mathbb{H}:=\{z: \operatorname{Im} z>0\}$, рассматривая функции как конечного, так и бесконечного порядка и оценивая величину исключительного множества. В случае субгармонических в $\mathbb{H}$ функций конечного порядка мы показываем, что существенно уточнить найденную аппроксимацию невозможно.

Используем основные факты и стандартные обозначения теории субгармонических функций [5]. Обозначим через $\mathscr{B}(r, u)$ максимум субгармонической в $\mathbb{H}$ функции $u(z)$ на множестве $\mathscr{D}(r):=\{z:|z-i r / 2| \leqslant r / 2,|z| \geqslant 1\}$, через $n\left(r, \mu_{u}\right)$ меру Рисса $\mu_{u}$ функции $u(z)$ множества $\mathscr{D}(r)$, буквами $C$ с индексами положительные постоянные, в скобках указьваем зависимость от параметров. Обозначим через $F M$ множество подмножеств $[1, \infty)$ конечной меры. Определим порядок $\rho[u]$ субгармонической в $\mathbb{H}$ функции $u$ формулой

$$
\rho[u]:=\limsup _{r \rightarrow \infty} \frac{\log \mathscr{B}(r, u)}{\log r} .
$$


Сформулируем результаты нашей работы.

ТЕОрема 1. Пусть субгармоническая в $\mathbb{H}_{1}:=\mathbb{H} \cup\{z:|z| \leqslant 1\}$ функиия и имеет конечный порядок $\rho=\rho[u]$ и число $\alpha>\rho+2$. Тогда существуют аналитическая в $\mathbb{H}_{1}$ функиия $f$, постоянная $C_{1}(\alpha)$ и множество $E$ такие, что выполнено неравенство

$$
|u(z)-\log | f(z)|| \leqslant C_{1}(\alpha)\left(\log ^{+}|z|+\log ^{+}\left(\frac{1}{\operatorname{Im} z}\right)+1\right), \quad z \notin E .
$$

Исключительное множество $E$, зависящее от функиий $u, f$ ичисла $\alpha$, содержится в обтединении кругов $D\left(z_{j}, r_{j}\right):=\left\{z:\left|z-z_{j}\right|<r_{j}\right\}, j \in \mathbb{N}, u$

$$
\sum_{z_{j} \notin \mathscr{D}(r)} r_{j}=o\left(r^{\rho-\alpha}\right), \quad r \rightarrow \infty \text {. }
$$

ТЕОрема 2. Пусть субгармоническая в $\mathbb{H}_{1}$ функиия и имеет бесконечный нижний порядок и число $\varepsilon>0$. Тогда существуют аналитическая в $\mathbb{H}_{1}$ функиия $f$, постоянная $C_{2}(\varepsilon)$ и мнохество $E$ такие, что для всех $z \notin E$ выполнено неравенство

$$
|u(z)-\log | f(z)|| \leqslant C_{2}(\varepsilon)\left(\log |z|+\log \left(\frac{1}{\operatorname{Im} z}\right)+\log \mathscr{B}\left(\frac{|z|^{2}}{\operatorname{Im} z}, u\right)\right) .
$$

Исключительное множество $E$, зависящее от функиий $u, f$ и числа в, содержится в обгединении кругов $D\left(z_{j}, r_{j}\right), j \in \mathbb{N}$, причем $(R=r+1 / \log \mathscr{B}(r, u))$

$$
\sum_{z_{j} \in \mathscr{D}(R) \backslash \mathscr{D}(r)} r_{j}=o\left(\mathscr{B}(r, u)^{-\varepsilon}\right), \quad r \rightarrow \infty, \quad r \notin S \in F M .
$$

Теорема 3. Пусть $\left\{a_{j}\right\}, j=1,2, \ldots,-$ - нли функции $f$, аналитической в $\mathbb{H}, u$ круги $D\left(a_{j},\left(\operatorname{Im} a_{j} /\left|a_{j}\right|^{2}\right)^{5 / 2}\right)$ не пересекаются. Тогда

$$
\limsup _{r \rightarrow \infty} \frac{\max \left\{|1 / \operatorname{Im} z-\log | f(z)||: z \in \mathscr{D}(r) \backslash \bigcup_{j} D\left(a_{j},\left(\operatorname{Im} a_{j} /\left|a_{j}\right|^{2}\right)^{5 / 2}\right)\right\}}{\log r} \geqslant \frac{1}{2} .
$$

Прокомментируем эти формулировки. Условие субгармоничности приближаемой функции также и в круге $\{z:|z| \leqslant 1\}$ чисто техническое; оно вызвано методом доказательства и в приложениях не обременительно. При доказательстве теоремы 2 используется новьй прием, который приводит к улучшению аналогичного известного результата для субгармонических в $\mathbb{C}$ функций бесконечного порядка (см. [6]).

Если субгармоническую в $\mathbb{H}$ функцию $1 / \operatorname{Im} z$ приближать аналитической (при этом в продолжении на круг $\{z:|z| \leqslant 1\}$ нет надобности), то по теореме 1 круги $D\left(z_{j}, r_{j}\right)$, объединение которых содержит исключительное множество, таковы, что

$$
r_{j} \asymp\left(\frac{\operatorname{Im} z_{j}}{\left|z_{j}\right|^{2}}\right)^{3} .
$$

Теорема 3 утверждает, что даже значительное увеличение исключительного множества не приводит к улучшению приближения. 
ДокАЗАТЕЛЬСТво тЕоРЕМЫ 1. Определим множество точек, $(\beta, s)$-нормальных относительно меры $\mu$, условием

$$
N(\beta, s, \mu):=\{z: \forall t \in(0, s) \quad(\mu(D(z, t)) \leqslant \beta t)\} .
$$

Будем использовать следующий результат Юлмухаметова [2, с. 275].

Теорема А. Пусть функиия и субгармоническая в неограниченной области $\Omega$. Тогда существует аналитическая в $\Omega$ функиия $f$, для всех $(\beta, s)$-нормальных точек относительно мер $\mu_{u} u \mu_{\log |f|}$ удовлетворяющая неравенству

$|u(z)-\log | f(z)|| \leqslant\left|\int_{D(0,2)} \log \right| z-\xi\left|d \mu_{u}(\xi)\right|+C_{3}|\log s|+C_{4} \log |z|+\beta s(|\log s|+1)+C_{5}$.

При этом постоянные $C_{3}, C_{4}, C_{5}$ не зависят от области $\Omega$ и от функиий $u, f$.

Как отмечает Юлмухаметов [2, с. 275], оценка сверху для функции $\log |f(z)|$, которая получается из (6), вьполняется для точек, $(\beta, s)$-нормальных только относительно меры $\mu_{u}$.

Согласно теореме А в условиях теоремы 1 существует аналитическая в $\mathbb{H}_{1}$ функция $f$, удовлетворяющая неравенству (1) для всех точек

$$
z \in N\left(\beta, s, \mu_{u}\right) \cap N\left(\beta, s, \mu_{\log |f|}\right)
$$

где

$$
\beta:=\left(\frac{|z|^{2}}{\operatorname{Im} z}\right)^{\alpha}, \quad s=\beta^{-1}, \quad \alpha>\rho+2 .
$$

Переходим к доказательству оценки (2) величины исключительного множества

$$
E=\mathbb{H}_{1} \backslash\left(N\left(\beta, s, \mu_{u}\right) \cap N\left(\beta, s, \mu_{\log |f|}\right)\right)
$$

(Отметим, что доказательство аналогичной оценки для случая плоскости в $[1$, c. 278-279] не полно.) Положим $\mu=\mu_{u}+\mu_{\log |f|}$. Далее будет доказано неравенство

$$
n(r, \mu) \leqslant C_{6} r^{\rho+1+\varepsilon} \text {. }
$$

В предположении, что (7) верно, оценим величину исключительного множества $E$. По определению точки, нормальной относительно меры, каждая точка $z \in E$ является центром круга $D(z, r(z))$ такого, что

$$
\mu(D(z, r(z))) \geqslant\left(\frac{|z|^{2}}{\operatorname{Im} z}\right)^{\alpha} r(z), \quad r(z) \in(0, s) .
$$

По теореме о покрытии [7, с. 246] из покрытия $\{D(z, r(z))\}$ множества $E$ можно выбрать не более чем счетное подпокрытие $\left\{D\left(z_{j}, r\left(z_{j}\right)\right)\right\}$ (далее обозначаем $\left.r_{j}:=r\left(z_{j}\right)\right)$, кратность которого не более 6 . Обозначим

$$
I_{n}:=\left\{j: z_{j} \in \mathscr{D}\left(2^{n+1}\right) \backslash \mathscr{D}\left(2^{n}\right)\right\}, \quad n \in \mathbb{N}
$$


Применяя оценку (8), имеем

$$
\sum_{j \in I_{n}} \mu\left(D\left(z_{j}, r_{j}\right)\right) \geqslant \sum_{j \in I_{n}} r_{j}\left(\frac{\left|z_{j}\right|^{2}}{\operatorname{Im} z_{j}}\right)^{\alpha} \geqslant \sum_{j \in I_{n}} r_{j} \min _{j}\left(\frac{\left|z_{j}\right|^{2}}{\operatorname{Im} z_{j}}\right)^{\alpha} \geqslant 2^{n \alpha} \sum_{j \in I_{n}} r_{j} .
$$

Последнее неравенство в (9) следует из того, что функция $|z|^{2} / \operatorname{Im} z=r$ на окружности $\{z:|z-i r / 2|=r / 2\}$.

С другой стороны, применив оценку (7), мы получаем

$$
\sum_{j \in I_{n}} \mu\left(D\left(z_{j}, r_{j}\right)\right) \leqslant 6 C_{6} 2^{(\rho+1+\varepsilon)(n+1)} .
$$

Сравнивая (9) и (10), находим, что

$$
\sum_{j \in I_{n}} r_{j} \leqslant 6 C_{6} 2^{\rho+1+\varepsilon} 2^{(\rho+1+\varepsilon-\alpha) n},
$$

а отсюда легко получается оценка (2). Отметим, что из оценки (11) следует, что исключительные круги $D\left(z_{j}, r_{j}\right)$, для которых $j \in I_{n}$, не покрывают дуг единичной окружности в $\mathscr{D}\left(2^{n+1}\right) \backslash \mathscr{D}\left(2^{n}\right)$, так как сумма длин указанных дуг является числом $\asymp 2^{-n}$, а $\alpha>\rho+2$. Осталось доказать (7). В этом месте мы используем формулу Левина [8, c. 22,41$]$, которую для удобства читателей процитируем.

Пусть $f$-мероморфная в $\mathbb{H}_{1}$ функция. Обозначим через $n(r, f)$ число полюсов функции $f$ (с учетом их кратностей), принадлежащих $\mathscr{D}(r)$. Положим

$$
\mathcal{N}(r, f):=\int_{1}^{\kappa(r)} \frac{n(t, f)}{t^{2}} d t, \quad \kappa(r):=\arcsin \left(r^{-1}\right) .
$$

Теорема В. Пусть функиия $f$, не равная тохдественно нулю, мероморфна в $\mathbb{H}_{1}$. Тогда выполнено равенство

$$
\mathcal{N}\left(r, \frac{1}{f}\right)-\mathcal{N}(r, f)=\frac{1}{2 \pi} \int_{\kappa(r)}^{\pi-\kappa(r)} \log \left|f\left(r e^{i \varphi} \sin \varphi\right)\right| \frac{d \varphi}{r \sin ^{2} \varphi}+Q(r, f),
$$

əde

$$
\begin{aligned}
Q(r, f) & =\frac{1}{2 \pi} \int_{\kappa(r)}^{\pi-\kappa(r)}\left(\log \left|f\left(e^{i \varphi}\right)\right|(-\sin \varphi)-\left.\left(\sin \varphi-\frac{1}{R}\right)\left(\log \left|f\left(r e^{i \varphi}\right)\right|\right)_{r}^{\prime}\right|_{r=1}\right) d \varphi \\
& =O(1)
\end{aligned}
$$

npu $r \rightarrow \infty$.

Заметим, что ограниченность $Q(r, f)$ в теореме В следует именно из мероморфности функции $f$ в круге $\{z:|z| \leqslant 1\}$. Из субгармонического варианта теоремы В получаем (при $\varepsilon>0)$

$$
\begin{aligned}
n\left(r, \mu_{u}\right) \frac{r}{4 r^{2}} & \leqslant n\left(r, \mu_{u}\right) \int_{r}^{2 r} \frac{d t}{t^{2}} \leqslant \int_{r}^{2 r} \frac{n(t) d t}{t^{2}} \leqslant \frac{1}{2 \pi} \int_{\kappa(2 r)}^{\pi-\kappa(2 r)} u\left(2 r e^{i \varphi} \sin \varphi\right) \frac{d \varphi}{2 r \sin ^{2} \varphi} \\
& \leqslant \frac{\mathscr{B}(2 r, u)}{2 \pi} \int_{\kappa(2 r)}^{\pi-\kappa(2 r)} \frac{d \varphi}{2 r \sin ^{2} \varphi} \leqslant C_{7} r^{\rho+1+\varepsilon},
\end{aligned}
$$


а отсюда неравенство

$$
n\left(r, \mu_{u}\right) \leqslant C_{8} r^{\rho+1+\varepsilon}
$$

Выше также было использовано определение порядка субгармонической в $\mathbb{H}_{1}$ функции $u$. Таким образом, для исключительного множества $E_{u}:=\mathbb{H}_{1} \backslash N\left(\beta, s, \mu_{u}\right)$ вьполняется (2). Как отмечено после формулировки теоремы А, функция $f$ удовлетворяет оценке сверху

$$
\log |f(z)| \leqslant u(z)+C_{1}(\alpha)\left(\log ^{+}|z|+\log ^{+} \frac{1}{\operatorname{Im} z}\right)
$$

вне $E_{u}$. Из (12) и доказательства неравенств $(9),(10)$ следует, что для любой точки $z \in \mathbb{H}$ существует окружность $\{w:|w-i r / 2|=r / 2\}$, где $r / 2 \in\left[|z|^{2} / \operatorname{Im} z,|2 z|^{2} / \operatorname{Im}(2 z)\right]$, все точки которой $(\beta, s)$-нормальны относительно меры $\mu_{u}$ и на которой, таким образом, выполняется неравенство (13). Опять применим теорему В и получим

$$
\begin{aligned}
\frac{n\left(r, \mu_{\log |f|}\right)}{4 r} & \leqslant \frac{1}{2 \pi} \int_{\kappa(2 r)}^{\pi-\kappa(2 r)} u\left(2 r e^{i \varphi} \sin \varphi\right) \frac{d \varphi}{2 r \sin ^{2} \varphi} \\
& \leqslant\left(\mathscr{B}(2 r, u)+C_{1}(\alpha)\left(\log ^{+}(2 r)+\log ^{+} \frac{1}{\kappa(2 r)}\right)\right) \frac{1}{2 \pi} \int_{\kappa(2 r)}^{\pi-\kappa(2 r)} \frac{d \varphi}{2 r \sin ^{2} \varphi} \\
& \leqslant C_{9}(\alpha) r^{\rho+\varepsilon} .
\end{aligned}
$$

Объединяя (12) и (14), получаем (7). Заметим, что при доказательстве соотношения (7) не возникает порочньй круг импликаций.

ДоКАЗАТЕЛЬСТВО ТЕОРЕМЫ 2. Оно в значительной мерепохоженапредыдущее доказательство, поэтому будем излагать кратко, отмечая новые моменты. Снова применим теорему А с $\beta=\mathscr{B}\left(|z|^{2} / \operatorname{Im} z, u\right)^{1+\varepsilon}, s=\beta^{-1}$ и отсюда получим (3).

Далее будет показано, что мера $\mu:=\mu_{u}+\mu_{\log |f|}$ удовлетворяет неравенству (при $0<3 \delta<\varepsilon)$

$$
n(r, \mu) \leqslant C_{10} \mathscr{B}(r, u)^{1+\delta}, \quad r \notin S \in F M .
$$

В предположении, что (15) вьполнено, докажем, что множество $E=\mathbb{H}_{1} \backslash N(\beta, s, \mu)$ является исключительным. Таким же образом, как и при доказательстве теоремы 1 , получаем, что существует не более чем счетное покрытие $E$ кругами $\left\{D\left(z_{j}, r_{j}\right)\right\}$, кратность которого не превышает 6 , обладающее следующим свойством:

$$
\mu\left(D\left(z_{j}, r_{j}\right)\right) \geqslant \mathscr{B}\left(\frac{\left|z_{j}\right|^{2}}{\operatorname{Im} z_{j}}, u\right)^{1+\varepsilon} r_{j}
$$

Положим $R=r+(\log \mathscr{B}(r, u))^{-1}$ и рассмотрим сумму

$$
\sum_{z_{j} \in \mathscr{D}(R) \backslash \mathscr{D}(r)} \mu\left(D\left(z_{j}, r_{j}\right)\right) \geqslant \sum_{z_{j} \in \mathscr{D}(R) \backslash \mathscr{D}(r)} \mathscr{B}(r, u)^{1+\varepsilon} r_{j}
$$

Вьше мы применили (16), а также использовали неубьвание функции $\mathscr{B}(r, u)$ и то обстоятельство, что при $z_{j} \in \mathscr{D}(R) \backslash \mathscr{D}(r)$ выполнены неравенства $r \leqslant\left|z_{j}\right|^{2} / \operatorname{Im} z_{j} \leqslant R$. Мы будем применять следующий вариант теоремы Бореля-Неванлинны [8, с. 121]. 
Теорема С. Пусть на $\left[r_{0}, \infty\right)$ задана непрерывная функция $v(r)$, неубывающая, стремящаяся $\kappa+\infty$ при $r \rightarrow \infty$, и число $\delta>0$. Тогда для всех $r \geqslant r_{0}$, кроме, возможно, множества конечной меры, выполняется

$$
v\left(r+\frac{1}{\log v(r)}\right)<v(r)^{1+\delta} .
$$

Теперь, учитьвая радиусы исключительных кругов и применяя теорему С, имеем

$$
\begin{aligned}
\sum_{z_{j} \in \mathscr{D}(R) \backslash \mathscr{D}(r)} \mu\left(D\left(z_{j}, r_{j}\right)\right) & \leqslant 6 \mu\left(\mathscr{D}\left(R+\frac{1}{\mathscr{B}(R, u)^{1+\varepsilon}}\right)\right) \\
& \leqslant 6 \mu\left(\mathscr{D}\left(R+\frac{1}{\log \mathscr{B}(R, u)}\right)\right) \leqslant 6 C_{10} \mathscr{B}(R, u)^{1+\delta} .
\end{aligned}
$$

Продолжим оценку (18):

$$
\sum_{z_{j} \in \mathscr{D}(R) \backslash \mathscr{D}(r)} \mu\left(D\left(z_{j}, r_{j}\right)\right) \leqslant C_{11} \mathscr{B}(r, u)^{(1+\delta)^{2}} \leqslant C_{11} \mathscr{B}(r, u)^{1+3 \delta}, \quad r \notin S \in F M .
$$

Из (17) и (19) следует (4). Осталось доказать (15). Из условия $\left.u(z) \leqslant \mathscr{B}|z|^{2} / \operatorname{Im} z, u\right)$ и теоремы В получаем

$$
\begin{aligned}
\frac{1}{2 \pi} \int_{\kappa(R)}^{\pi-\kappa(R)} \frac{d \varphi}{R \sin ^{2} \varphi} \mathscr{B}(R, u) & \geqslant \frac{1}{2 \pi} \int_{\kappa(R)}^{\pi-\kappa(R)} u\left(R e^{i \varphi} \sin \varphi\right) \frac{d \varphi}{R \sin ^{2} \varphi} \\
& \geqslant C_{12} \int_{r}^{R} \frac{n\left(t, \mu_{u}\right)}{t^{2}} d t \geqslant C_{12} n\left(r, \mu_{u}\right)\left(\frac{1}{r}-\frac{1}{R}\right) .
\end{aligned}
$$

Отсюда следует, что

$$
n\left(r, \mu_{u}\right) \leqslant C_{13} \mathscr{B}(r, u)^{1+\delta / 2} r^{2} \log \mathscr{B}(r, u) \leqslant \mathscr{B}(r, u)^{1+\delta}, \quad r \notin S \in F M .
$$

Подобно доказательству теоремы 1 показываем, что из оценки (20) следует (4) для множества $E_{u}=\mathbb{H}_{1} \backslash N\left(\beta, s, \mu_{u}\right)$. Согласно замечанию к теореме $\mathrm{A}$ аналитическая в $\mathbb{H}_{1}$ функция $f$, построенная по этой теореме, удовлетворяет неравенству

$$
\begin{aligned}
\log |f(z)| & \leqslant u(z)+C_{2}(\varepsilon)\left(\log ^{+} \mathscr{B}\left(\frac{|z|^{2}}{\operatorname{Im} z}, u\right)+\log ^{+}|z|+\log ^{+} \frac{1}{\operatorname{Im} z}\right) \\
& \leqslant 2 \mathscr{B}\left(\frac{|z|^{2}}{\operatorname{Im} z}, u\right)+C_{2}(\varepsilon) \log ^{+} \frac{|z|}{\operatorname{Im} z}, \quad z \notin E_{u} .
\end{aligned}
$$

Из оценки (4) для исключительного множества $E_{u}$ следует, что для любой точки $z \in \mathbb{H},|z|>1$, существует окружность

$$
\left\{w:\left|w-\frac{i t}{2}\right|=\frac{t}{2}\right\}, \quad t \in[r, R]
$$


где $r=|z|^{2} / \operatorname{Im} z, R=r+(\log \mathscr{B}(r, u))^{-1}$, все точки которой $(\beta, s)$-нормальны относительно меры $\mu_{u}$. Применяя принцип максимума для субгармонических функций и учитьвая неубьвание $\mathscr{B}(r, u)$, получаем неравенство

$$
\log |f(z)| \leqslant 2 \mathscr{B}(R, u)+C_{2}(\varepsilon) \log ^{+} \frac{|z|}{\operatorname{Im} z} \leqslant 2 \mathscr{B}(r, u)^{1+\delta / 2}+C_{2}(\varepsilon) \log ^{+} \frac{|z|}{\operatorname{Im} z}
$$

для всех $z \in \mathbb{H}$, кроме $r=|z|^{2} / \operatorname{Im} z \in S \in F M$. Снова из теоремы В вьводим

$$
\begin{aligned}
n\left(r, \mu_{\log |f|}\right)\left(\frac{1}{r}-\frac{1}{t}\right) & \leqslant \frac{1}{2 \pi} \int_{\kappa(t)}^{\pi-\kappa(t)} u\left(t e^{i \varphi} \sin \varphi\right) \frac{d \varphi}{t \sin ^{2} \varphi} \\
& \leqslant C_{14}\left(2 \mathscr{B}(R, u)+C_{2}(\varepsilon) \log ^{+} \frac{|z|}{\operatorname{Im} z}\right)
\end{aligned}
$$

а отсюда и из бесконечности нижнего порядка функции $u$ следует

$$
n\left(r, \mu_{\log |f|}\right) \leqslant C_{15} \mathscr{B}(r, u)^{1+\delta}, \quad r \notin S \in F M
$$

Сочетая $(20)$ и (22), имеем (15).

ДоКАЗАТЕЛЬСТво ТЕОРЕмы 3. Отметим, что функция $v(z)=1 / \operatorname{Im} z$ субгармоническая в $\mathbb{H}$ порядка 1 нормального типа. Обозначим через $v_{R}(z)=2 R+\sqrt{R}-$ $R(R+\sqrt{R}) \operatorname{Im} z$ ее наименьшую гармоническую мажоранту $[5, \S 2.4]$ в полосе

$$
\left\{z: \frac{1}{R+\sqrt{R}} \leqslant \operatorname{Im} z \leqslant \frac{1}{R}\right\}
$$

т.е. $v(z)=v_{R}(z)$ на граничных прямых и $v(z)<v_{R}(z)$ внутри полосы. Оказывается, и это является одним из ключевых моментов доказательства, что

$$
\max \left\{v_{R}(z)-v(z): \frac{1}{R+\sqrt{R}} \leqslant \operatorname{Im} z \leqslant \frac{1}{R}\right\} \leqslant \frac{1}{4} .
$$

Соотношение (23) легко проверить стандартными средствами анализа, а чтобы избежать рутинных вькладок, для этого можно употребить один из математических пакетов, например, Maple (cм. www . maplesoft . com).

Далее, пусть $f(a)=0, \operatorname{Im} a<1$. Обозначим через Г кривую, составленную из дуг окружностей

$$
S\left(a,\left(\frac{\operatorname{Im} a}{|a|^{2}}\right)^{3 / 2}\right):=\left\{z:|z-a|=\left(\frac{\operatorname{Im} a}{|a|^{2}}\right)^{3 / 2}\right\}
$$

и из дуг окружностей

$$
S\left(a_{n},\left(\frac{\operatorname{Im} a_{n}}{\left|a_{n}\right|^{2}}\right)^{5 / 2}\right):=\left\{z:|z-a|=\left(\frac{\operatorname{Im} a_{n}}{\left|a_{n}\right|^{2}}\right)^{5 / 2}\right\}
$$

пересекающихся с ней, таким образом, чтобы кривая Г была простой, замкнутой, содержащей внутри точку $a$ и лежащей внутри $S\left(a,\left(\operatorname{Im} a /|a|^{2}\right)^{3 / 2}\right)$. 
Пусть $h_{v}$ и $h_{\log |f|}-$ функции, гармонические внутри $Г$ и равные на $Г$ соответственно $v(z)$ и $\log |f(z)|$.

Предположим, что утверждение теоремы 3 не верно и при некотором $\sigma_{1}<1 / 2$ для всех $r>r_{0}$ вьполняется неравенство

$$
\max \left\{|v(z)-\log | f(z)||: z \in \mathscr{D}(r),\left|z-a_{n}\right| \geqslant\left(\frac{\operatorname{Im} a_{n}}{\left|a_{n}\right|^{2}}\right)^{5 / 2}\right\} \leqslant \sigma_{1} \log r
$$

Тогда при достаточно малом $\operatorname{Im} a /|a|^{2}$ для всех точек из int $\Gamma$ - внутренности $\Gamma$ - имеем

$$
\left|h_{v}(z)-v(z)\right| \leqslant \frac{1}{4}
$$

Действительно,

$$
\operatorname{int} \Gamma \subset \operatorname{int} S \subset\left\{z: \frac{1}{R+\sqrt{R}} \leqslant \operatorname{Im} z \leqslant \frac{1}{R}\right\}
$$

c $R=(2 \operatorname{Im} a)^{-1}$.

Из принципа максимума для гармонических функций следует неравенство (при $\sigma_{1}<$ $\left.\sigma_{2}<1 / 2\right)$

$$
\left|h_{\log |f|}-h_{v}(z)\right| \leqslant \sigma_{2} \log \frac{|z|^{2}}{\operatorname{Im} z}, \quad z \in \operatorname{int} \Gamma .
$$

В самом деле, на Г оно выполняется по определению функций $h_{\log |f|}, h_{v}$ и $(24)$. Теперь

$$
\begin{aligned}
\left|h_{\log |f|}(z)-h_{v}(z)\right| & \leqslant \max \left\{\left|h_{\log |f|}(z)-h_{v}(z)\right|: z \in \Gamma\right\} \\
& =\left|h_{\log |f|}\left(z_{0}\right)-h_{v}\left(z_{0}\right)\right| \leqslant \sigma_{2} \log \frac{\left|z_{0}\right|^{2}}{\operatorname{Im} z_{0}}, \quad z_{0} \in \Gamma .
\end{aligned}
$$

Отсюда и из неравенства $\left|z_{0}\right|^{2} / \operatorname{Im} z_{0} \leqslant 2|z|^{2} / \operatorname{Im} z$ получаем (26). Сочетая (24)-(26), получаем оценку

$$
\begin{aligned}
\left|h_{\log |f|}(z)-\log \right| f(z)|| & \leqslant\left|h_{\log |f|}(z)-h_{v}(z)\right|+\left|h_{v}(z)-v(z)\right|+|v(z)-\log | f(z)|| \\
& \leqslant \sigma_{3} \log \frac{|z|^{2}}{\operatorname{Im} z}, \quad z \in \operatorname{int} \Gamma
\end{aligned}
$$

c $\sigma_{1}+\sigma_{2}<\sigma_{3}<1$. Согласно формуле Пуассона-Иенсена [5, §3.7], примененной к $\log |f(z)|$,

$$
\log |f(z)|=h_{\log |f|}(z)-\sum_{a_{n} \in \operatorname{int} \Gamma} g\left(z, a_{n}, \operatorname{int} \Gamma\right)
$$

где $g\left(z, a_{n}, \operatorname{int} \Gamma\right)$ - функция Грина области int $\Gamma$ с полюсом в $a_{n}[5, \S 1.5]$, имеем

$$
\left|h_{\log |f|}(z)-\log \right| f(z)||=\mid \sum_{a_{n} \in \operatorname{int} \Gamma} g\left(z, a_{n}, \text { int } \Gamma\right) \mid
$$


Используя известные свойства функции Грина односвязной области [8, с. 11] и вариационные свойства конформных отображений [9, с. 359], приходим к неравенству

$$
\begin{aligned}
\left|h_{\log |f|}(z)-\log \right| f(z)|| & \geqslant g(z, a, \operatorname{int} \Gamma) \\
& \geqslant g\left(z, a, D\left(a,\left(\frac{\operatorname{Im} a}{|a|^{2}}\right)^{3 / 2}\right)\right)=\log \frac{\left(\operatorname{Im} a /|a|^{2}\right)^{3 / 2}}{|z-a|} .
\end{aligned}
$$

Выберем точку $z$ так, что

$$
\operatorname{Im} z=\operatorname{Im} a, \quad|z-a|=\left(\frac{\operatorname{Im} a}{|a|^{2}}\right)^{5 / 2}, \quad|z|<|a|
$$

Подставив эту точку в (29), мы получим

$$
\left|h_{\log |f|}(z)-\log \right| f(z)|| \geqslant \log \frac{|a|^{2}}{\operatorname{Im} a}>\log \frac{|z|^{2}}{\operatorname{Im} z}
$$

а это противоречит (27).

Обоснуем теперь существование корней $a$ аналитической функции $f$ с каким угодно большим значением $|a|^{2} / \operatorname{Im} a$; это утверждение использовалось вьше. Считающая функция $n(r, v)$ субгармонической в $\mathbb{H}$ функции $v(z)=1 / \operatorname{Im} z$ имеет следующую асимптотику:

$n(r, v)=\frac{1}{2 \pi} \int_{\mathscr{D}(r)} \Delta v d x d y \sim \frac{2 r^{2}}{3 \pi}+\frac{-6+6 \log 2+6 \log r}{6 \pi}-\frac{5}{96 \pi r^{4}}+O\left(r^{-6}\right), \quad r \rightarrow \infty$.

Указанная асимптотика найдена с помощью пакета Maple.

Пусть $n(r, \log |f|)=O(1), r \rightarrow \infty$. Запишем формулу Левина

$$
\begin{aligned}
& \int_{1}^{r} \frac{n(t, \log |f|)-n(t, v)}{t^{2}} d t \\
& \quad=\frac{1}{2 \pi} \int_{\kappa(r)}^{\pi-\kappa(r)} \frac{\log \left|f\left(r e^{i \varphi}\right)\right|-v\left(r e^{i \varphi} \sin \varphi\right)}{r \sin ^{2} \varphi} d \varphi+O(1), \quad r \rightarrow \infty .
\end{aligned}
$$

Тогда левая сторона (30) есть $\asymp r, r \rightarrow \infty$, а правая согласно $(24)$ равна $O(\log r), r \rightarrow \infty$; получили противоречие.

Выражаю признательность члену-корреспонденту НАН Украины профессору И.В. Островскому за внимание к работе, профессору В.Я. Эйдерману за полезньй совет и участникам львовского семинара по комплексному анализу за обсуждение результатов. 


\section{СПИСОК ЦИТИРОВАННОЙ ЛИТЕРАТУРЫ}

[1] Азарин В.С. О лучах вполне регулярного роста целой функции // Матем. сб. 1969. T. 79 (121). № 4 (8). C. 463-476.

[2] Юлмухаметов Р.С.Аппроксимация субгармонических функций // Anal. Math. 1985. V. 11. № 3. P. 257-282.

[3] Drasin D. Approximation of subharmonic functions with applications // Approximation, Complex Analysis, and Potential Theory. Proc. of the NATO ASI (Montreal, Canada, 3-14 July 2000). Dordrecht-Boston-London: Kluwer Acad. Publ., 2001. P. 163-189.

[4] Chyzhykov I. Approximation of subharmonic functions of slow growth // Матем. физ., анализ, геом. 2002. Т. 9. № 3. С. 509-520.

[5] Hayman W. K., Kennedy P. B. Subharmonic Functions. V. 1. London-New York-San Francisco: Academic Press, 1976.

[6] Гирнык М.А. О приближении субгармонической функции бесконечного порядка логарифмом модуля целой функции // Матем. заметки. 1991. Т. 50. № 4. С. 57-60.

[7] Ландкоф Н. С. Основы современной теории потенциала. М.: Наука, 1966.

[8] Гольдберг А. А., Островский И. В. Распределение значений мероморфных функций. М.: Наука, 1970.

[9] Лаврентьев М. А., Шабат Б. В. Методы теории функций комплексного переменного. М.: Наука, 1973. 